\title{
Energy-consistent entrainment relations for jets and plumes
}

\author{
Maarten van Reeuwijk $k^{1, \dagger}$ and John Craske ${ }^{1}$ \\ ${ }^{1}$ Department of Civil and Environmental Engineering, Imperial College London, London SW7 2AZ, UK
}

(Received 30 March 2015; revised 20 July 2015; accepted 7 September 2015;

first published online 8 October 2015)

We discuss energetic restrictions on the entrainment coefficient $\alpha$ for axisymmetric jets and plumes. The resulting entrainment relation includes contributions from the mean flow, turbulence and pressure, fundamentally linking $\alpha$ to the production of turbulence kinetic energy, the plume Richardson number $R i$ and the profile coefficients associated with the shape of the buoyancy and velocity profiles. This entrainment relation generalises the work by Kaminski et al. (J. Fluid Mech., vol. 526, 2005, pp. 361-376) and Fox (J. Geophys. Res., vol. 75, 1970, pp. 6818-6835). The energetic viewpoint provides a unified framework with which to analyse the classical entrainment models implied by the plume theories of Morton et al. (Proc. R. Soc. Lond. A, vol. 234, 1955, pp. 1-23) and Priestley \& Ball (Q. J. R. Meteorol. Soc., vol. 81, 1954, pp. 144-157). Data for pure jets and plumes in unstratified environments indicate that to first order the physics is captured by the Priestley and Ball entrainment model, implying that (1) the profile coefficient associated with the production of turbulence kinetic energy has approximately the same value for pure plumes and jets, (2) the value of $\alpha$ for a pure plume is roughly a factor of 5/3 larger than for a jet and (3) the enhanced entrainment coefficient in plumes is primarily associated with the behaviour of the mean flow and not with buoyancy-enhanced turbulence. Theoretical suggestions are made on how entrainment can be systematically studied by creating constant- $R i$ flows in a numerical simulation or laboratory experiment.

Key words: free shear layers, wakes/jets, plumes/thermals

\section{Introduction}

The entrainment hypothesis is the standard turbulence closure used in integral descriptions of turbulent jets and plumes. It links the entrainment velocity, the rate at which ambient fluid is entrained into the plume, to a typical velocity inside the plume by a single coefficient of proportionality $\alpha$, the entrainment coefficient. For axisymmetric releases, the entrainment hypothesis takes the form (Turner 1986)

$$
-(r u)_{r=\infty}=\alpha \hat{r} \hat{w}
$$

where $r$ and $u$ denote the radial direction and radial velocity, and $\hat{r}$ and $\hat{w}$ are the characteristic plume radius and velocity respectively. The entrainment coefficient $\alpha$ is generally the only parameter representing the effect that turbulence has on the mean

$†$ Email address for correspondence: m.vanreeuwijk@imperial.ac.uk 
flow, and it is remarkable that the behaviour of a flow as complex as a turbulent plume can be captured by a closure as simple as (1.1). In this respect it could be regarded as the free-shear equivalent of the quadratic friction relation for wall-bounded flows under fully rough conditions, which links the wall shear stress to the free-stream velocity via a single friction coefficient.

The value of $\alpha$ is subject to significant variability: typical values for the 'top-hat' entrainment coefficient are in the range $0.065<\alpha<0.080$ in jets and $0.10<\alpha<0.16$ in pure plumes (Fischer et al. 1979; Carazzo, Kaminski \& Tait 2006). Here, the term 'top-hat' refers to a description that assumes a uniform value of profiles of velocity and buoyancy over a finite radius. The flux-balance parameter $\Gamma$ (Morton 1959), which will be rigorously defined in $\S 2$, allows one to distinguish between jets and pure plumes. In neutrally stratified environments, vertically (denoted $z$ ) oriented flows evolve in such a way that $\Gamma(z)$ either remains constant or approaches an asymptotic limit. For a pure jet, which has no buoyancy, $\Gamma(z)=0$. Plumes that have an excess of momentum at their source are called 'forced', and $\Gamma$ is in the range $0<\Gamma<1$; plumes that have a deficit of momentum at their source are called 'lazy', and have $\Gamma>1$ (Hunt \& Kaye 2005). Both forced and lazy plumes will transition to a pure plume $(\Gamma=1)$ as the flow develops. A review of jets and plumes can be found in, e.g., Hunt \& van den Bremer (2011).

There is significant variation in $\alpha$ between different experiments, as is evident from the large range of observed values. This can be attributed to differences in experimental set-ups, experimental error and uncertainty (Turner 1986; Kaminski, Tait \& Carazzo 2005), and also source conditions (George 1989; Redford, Castro \& Coleman 2012). However, it is evident that there is a systematic difference between the reported value of $\alpha$ for jets and plumes, which suggests a dependence of $\alpha$ on $\Gamma$.

In this paper we consider restrictions imposed upon the entrainment coefficient $\alpha$ by the equation for the mean kinetic energy. We will refer to this restriction as an entrainment relation hereafter. The entrainment relation couples $\alpha$ to various physical processes such as turbulence production and buoyancy effects. We will refer to entrainment models as the closed relations that are obtained once the various coefficients in the entrainment relation are parameterised. The aim of this paper is to provide a hierarchy of energy-consistent entrainment relations that can be used either in a diagnostic mode, clarifying the physics of turbulent entrainment, or in a prognostic mode, leading to entrainment models that can be used for predictive purposes.

To date, no distinction has been made between an entrainment relation and an entrainment model, perhaps because much of the discussion in the literature has focused on the reconciliation of the plume theories of Morton, Taylor \& Turner (1956, MTT) and Priestley \& Ball (1955, PB). These theories are obtained by integrating the axisymmetric Reynolds-averaged Navier-Stokes equations across a plane perpendicular to the mean-flow direction, resulting in a system of coupled ordinary differential equations (ODEs) in terms of integral quantities. The MTT plume equations comprise three ODEs in terms of the volume flux $Q$, the momentum flux $M$ and the buoyancy flux $F$. Entrainment into the plume is quantified by a constant parameter $\alpha$ (the entrainment coefficient) which features in the volume conservation equation. The PB plume equations consist of three coupled ODEs for $M, F$ and the flux of mean kinetic energy $E$. The PB plume equations rely on a parameterisation of the production of turbulence kinetic energy, or equivalently the Reynolds stress.

A lucid description of how the two models are related was provided by Fox (1970); by simultaneously considering the conservation equations of volume, momentum, 
buoyancy and mean kinetic energy, he linked the two models and was the first to highlight the constraints imposed on $\alpha$ by the conservation equation for mean kinetic energy, i.e. the entrainment relation. The analysis was restricted to fully self-similar profiles (i.e. the far field), and his model took into account the possibility of the velocity and buoyancy profiles having different widths. Apart from deriving the far-field entrainment relation, Fox derived the PB entrainment model, given by (Fox 1970; List \& Imberger 1973)

$$
\alpha=\alpha_{j}+\left(\alpha_{p}-\alpha_{j}\right) \Gamma,
$$

where $\alpha_{j}$ and $\alpha_{p}$ are the entrainment coefficients for a pure jet and a pure plume respectively. A few years later List \& Imberger (1973), starting from the observation that jets and plumes spread at practically the same rate (see also List 1982), derived an entrainment model compatible with (1.2). This is indeed consistent - solutions to the PB model are straight-sided, with identical spreading rate regardless of the value of $\Gamma$, including forced and lazy plumes (Priestley \& Ball 1955, see also §5). The PB entrainment model provides predictions for $\alpha$ that are in reasonably good agreement with laboratory experiments on forced plumes in an unstratified ambient (Wang \& Law 2002; Matulka et al. 2014; Ezzamel, Salizzoni \& Hunt 2015).

In a response to Fox's analysis, Morton (1971) highlighted that for a plume rising in a linearly stratified ambient, both models were equally appropriate; however, near the source and the neutral buoyancy level the MTT approach was preferable because it was able to predict plume necking and spreading respectively. Here, necking refers to the observation that lazy plumes tend to contract relatively close to the source (see, e.g., Fannelop \& Webber 2003), and spreading refers to the widening of a plume once it reaches a location where the buoyancy of the environment is identical to that of the plume (the neutral buoyancy level). Indeed, a problem with (1.2) in stratified environments is that it admits a sign change in $\alpha$, which motivated the empirical model for the entrainment coefficient proposed by Fischer et al. (1979, p. 371). More sophisticated among attempts to model entrainment in plumes is the approach of Telford (1966). Recognising that the assumption of self-similarity is often violated in the context of atmospheric convection, Telford (1966) suggests that the entrainment coefficient is proportional to the turbulence intensity, and solves an additional equation for the turbulence kinetic energy.

Kaminski et al. (2005) should be credited for reintroducing energy-based entrainment relations to the plume literature. Indeed, they built on the foundations laid by Fox (1970), and extended the analysis by relaxing the assumptions regarding the radial profile dependences. This led to an entrainment relation valid for both the near field and the far field, which exposed the physics of entrainment in terms of leading-order quantities. While the main focus of Kaminski et al. (2005) was on turbulent fountains, they argued that the ratio of the width of the velocity profile to the buoyancy (reduced gravity) profile, denoted $\lambda$, could help to reconcile the spread in $\alpha$ for pure plumes. In particular, they showed evidence that small variations of $\lambda$ in the vertical direction had an effect on entrainment, a process they termed similarity drift. A disadvantage of their derivation is the use of top-hat scales for $\hat{r}$ and $\hat{w}$ that depend on the buoyancy flux, which is not standard procedure and is not a logical extension of the scales one defines in jets. This greatly complicates the use of their entrainment relation, as the relation between $\alpha$ and the Kaminski entrainment coefficient $\alpha_{e}$ is not trivial (see appendix A for details). The framework was further extended with turbulence and pressure contributions by Ezzamel et al. (2015), who provide an entrainment relation directly in terms of the Gaussian entrainment coefficient $\alpha_{G}$. They remark that similarity drift does not feature in $\alpha_{G}$ in the same way as in $\alpha_{e}$ (see also $\S 3$ ), which, as noted above, is a result of the different scale definitions employed. 
Consideration of a mean energy budget becomes crucial once the restriction of a steady state is lifted. This is because the various profile coefficients characterising the mean and turbulent profiles of velocity and buoyancy play an independent role in defining the structure of the governing integral equations (Craske \& van Reeuwijk $2015 a, b)$. Moreover, a mean kinetic energy equation for unsteady jets and plumes can be readily obtained without significant approximation, thereby circumventing the difficulties that are associated with obtaining a conservation equation for the volume of the plume (see, e.g., Scase \& Hewitt 2012). Indeed, due to the underlying assumptions, one finds significant differences between existing unsteady plume models (e.g. those of Delichatsios 1979; Yu 1990; Vul'fson \& Borodin 2001; Scase et al. 2006), whose properties and consistency can be checked with respect to an overarching framework by appealing to the mean energy equation (Craske \& van Reeuwijk 2015b). In the context of entrainment, the use of a momentum-energy framework allows one to deduce that under certain conditions both unsteady jets and plumes remain straight-sided (Craske 2015; Craske \& van Reeuwijk 2015b), and allowed Craske \& van Reeuwijk (2015a) to obtain a decomposition of the entrainment coefficient for jets, similar to that of Kaminski et al. (2005), but with the important distinction that (1) both turbulence and pressure contributions are included and (2) standard (top-hat) definitions for radius and velocity scales are used.

In $\S 2$, we adapt the momentum-energy framework developed in Craske \& van Reeuwijk $(2015 a, b)$ to include buoyancy. An entrainment relation that ensures compatibility between volume and energy conservation is derived in $\S 3$, after which a hierarchy of entrainment relations will be provided which naturally accommodate the entrainment relations of Kaminski et al. (2005) and Fox (1970). The implications of the entrainment relation and a physical interpretation are provided in $\S 4$. The entrainment models underlying the MTT and PB theories are discussed in $\S 5$ and an investigation into an appropriate entrainment model for pure jets and pure plumes in an unstratified environment is undertaken in $\S 6$. One of the key requirements in order to systematically study turbulent entrainment is to maintain a constant plume Richardson number $R i$ as the plume ascends; $\$ 7$ discusses possibilities of how a constant $R i$ may be achieved. Concluding remarks are made in $\S 8$.

\section{Governing equations}

Consider a round high-Reynolds-number turbulent plume orientated in the vertical (z) direction whose flow is statistically axisymmetric. We consider Reynolds-averaged conservation equations for volume, streamwise momentum and buoyancy. Adopting the Boussinesq approximation and assuming high-Reynolds-number flow, the governing equations are

$$
\begin{gathered}
\frac{1}{r} \frac{\partial}{\partial r}(r \bar{u})+\frac{\partial \bar{w}}{\partial z}=0, \\
\frac{1}{r} \frac{\partial}{\partial r}\left(r \bar{u} \bar{w}+r \overline{u^{\prime} w^{\prime}}\right)+\frac{\partial}{\partial z}\left(\bar{w}^{2}+\overline{w^{\prime 2}}\right)=-\frac{\partial \bar{p}}{\partial z}+\bar{b}, \\
\frac{1}{r} \frac{\partial}{\partial r}\left(r \bar{u} \bar{b}+r \overline{u^{\prime} b^{\prime}}\right)+\frac{\partial}{\partial z}\left(\bar{w} \bar{b}+\overline{w^{\prime} b^{\prime}}\right)=-N^{2} \bar{w} .
\end{gathered}
$$

Here, the mean velocity components $(\bar{u}, \bar{w})$ correspond to the directions $(r, z)$ respectively, $\bar{p}$ denotes the kinematic pressure from which the hydrostatic pressure field resulting from the environmental density $\rho_{e}(z)$ has been subtracted, and 
$\bar{b}=g\left(\rho_{e}-\bar{\rho}\right) / \rho_{0}$ is the buoyancy for which $\rho_{0}$ is a reference density. The buoyancy frequency is defined as $N^{2}(z)=-\left(g / \rho_{0}\right)\left(\mathrm{d} \rho_{e} / \mathrm{d} z\right)$.

By multiplying (2.2) with $2 \bar{w}$, we obtain after some manipulation

$$
\begin{aligned}
& \frac{1}{r} \frac{\partial}{\partial r}\left(r \bar{u} \bar{w}^{2}+2 r \overline{u^{\prime} w^{\prime}} \bar{w}\right)+\frac{\partial}{\partial z}\left(\bar{w}^{3}+2 \overline{w^{\prime 2}} \bar{w}+2 \bar{p} \bar{w}\right) \\
& \quad=2 \overline{u^{\prime} w^{\prime}} \frac{\partial \bar{w}}{\partial r}+2 \overline{w^{\prime 2}} \frac{\partial \bar{w}}{\partial z}+2 \bar{p} \frac{\partial \bar{w}}{\partial z}+2 \bar{w} \bar{b} .
\end{aligned}
$$

This equation will be referred to as the equation of mean kinetic energy, since the typical scale for the mean radial velocity is smaller than the streamwise velocity by a factor $\alpha$, implying that the mean kinetic energy is dominated by $\bar{w}^{2}$. The first two terms of (2.4) represent the radial and vertical transport terms, each containing mean and turbulent flux contributions. The term $2 \overline{u^{\prime} w^{\prime}}(\partial \bar{w} / \partial r)+2 \overline{w^{\prime 2}}(\partial \bar{w} / \partial z)$ is associated with the production of turbulence kinetic energy and forms a sink term in the equation for mean kinetic energy. The term $2 \bar{p}(\partial \bar{w} / \partial z)$ is a pressure redistribution term and $2 \bar{w} \bar{b}$ represents the production of mean kinetic energy due to buoyancy. We note that the absence of viscous terms in the equation for mean kinetic energy does not imply that viscous dissipation is neglected altogether. While the viscous dissipation associated with the mean-flow components is indeed negligible at high Reynolds number $(R e)$ (see, e.g., Tennekes \& Lumley 1972), the component associated with the turbulence cannot be neglected. The latter dissipation rate is crucial in the balance of turbulence kinetic energy, as it is of the same order of magnitude as the turbulence production term (Shabbir \& George 1994). However, the balance of turbulence kinetic energy is not considered in this paper, and viscous effects are thus entirely absent from the mathematical description of the mean flow.

The volume flux $Q$, momentum flux $M$, integral buoyancy $B$ and buoyancy flux $F$ are defined as

$$
Q \equiv 2 \int_{0}^{\infty} \bar{w} r \mathrm{~d} r, \quad M \equiv 2 \int_{0}^{\infty} \bar{w}^{2} r \mathrm{~d} r, \quad B \equiv 2 \int_{0}^{\infty} \bar{b} r \mathrm{~d} r, \quad F \equiv 2 \int_{0}^{\infty} \bar{w} \bar{b} r \mathrm{~d} r .
$$

These integral quantities can be used to define a characteristic plume width, velocity and buoyancy respectively:

$$
r_{m} \equiv \frac{Q}{M^{1 / 2}}, \quad w_{m} \equiv \frac{M}{Q}, \quad b_{m} \equiv \frac{B M}{Q^{2}} .
$$

These scales are consistent with top-hat variables; however, it should be noted that the analysis below does not make a priori assumptions regarding the profile shape.

Integration of (2.1)-(2.4) over $r$ results in

$$
\begin{gathered}
\frac{\mathrm{d} Q}{\mathrm{~d} z}=2 \alpha M^{1 / 2}, \\
\frac{\mathrm{d}}{\mathrm{d} z}\left(\beta_{g} M\right)=\frac{F Q}{\theta_{m} M}, \\
\frac{\mathrm{d}}{\mathrm{d} z}\left(\frac{\theta_{g}}{\theta_{m}} F\right)=-N^{2} Q, \\
\frac{\mathrm{d}}{\mathrm{d} z}\left(\gamma_{g} \frac{M^{2}}{Q}\right)=\delta_{g} \frac{M^{5 / 2}}{Q^{2}}+2 F .
\end{gathered}
$$

The right-hand side of the volume conservation equation (2.7) was obtained by making use of the entrainment hypothesis (1.1) using the characteristic scales defined 
in $(2.6 a-c)$. The parameters $\beta, \gamma, \theta$ and $\delta$ in (2.7)-(2.10) are profile coefficients associated with the dimensionless momentum flux, energy flux, buoyancy flux and turbulence production respectively. Consistent with Craske \& van Reeuwijk (2015a), the gross value of a profile coefficient, e.g. $\gamma_{g}$, is composed of contributions from the mean flow, turbulence and pressure, i.e. $\gamma_{g}=\gamma_{m}+\gamma_{f}+\gamma_{p}$. Explicitly, the profile coefficients are defined as

$$
\left.\begin{array}{c}
\beta_{m} \equiv \frac{M}{w_{m}^{2} r_{m}^{2}} \equiv 1, \quad \beta_{f} \equiv \frac{2}{w_{m}^{2} r_{m}^{2}} \int_{0}^{\infty} \overline{w^{\prime 2}} r \mathrm{~d} r, \quad \beta_{p} \equiv \frac{2}{w_{m}^{2} r_{m}^{2}} \int_{0}^{\infty} \bar{p} r \mathrm{~d} r, \\
\gamma_{m} \equiv \frac{2}{w_{m}^{3} r_{m}^{2}} \int_{0}^{\infty} \bar{w}^{3} r \mathrm{~d} r, \quad \gamma_{f} \equiv \frac{4}{w_{m}^{3} r_{m}^{2}} \int_{0}^{\infty} \bar{w} \overline{w^{\prime 2}} r \mathrm{~d} r, \quad \gamma_{p} \equiv \frac{4}{w_{m}^{3} r_{m}^{2}} \int_{0}^{\infty} \bar{w} \bar{p} r \mathrm{~d} r, \\
\delta_{m} \equiv \frac{4}{w_{m}^{3} r_{m}} \int_{0}^{\infty} \overline{w^{\prime} u^{\prime}} \frac{\mathrm{d} \bar{w}}{\mathrm{~d} r} r \mathrm{~d} r, \quad \delta_{f} \equiv \frac{4}{w_{m}^{3} r_{m}} \int_{0}^{\infty} \overline{w^{\prime 2}} \frac{\mathrm{d} \bar{w}}{\mathrm{~d} z} r \mathrm{~d} r, \quad \delta_{p} \equiv \frac{4}{w_{m}^{3} r_{m}} \int_{0}^{\infty} \bar{p} \frac{\mathrm{d} \bar{w}}{\mathrm{~d} z} \mathrm{~d} r, \\
\theta_{m} \equiv \frac{F}{w_{m} b_{m} r_{m}^{2}}, \quad \theta_{f} \equiv \frac{2}{w_{m} b_{m} r_{m}^{2}} \int_{0}^{\infty} \overline{w^{\prime} b^{\prime} r} \mathrm{~d} r .
\end{array}\right\}
$$

It should be noted that the definition of $\theta_{m}$ provides a fundamental relation between the integral buoyancy $B$ and the buoyancy flux $F$ as $B=F Q /\left(\theta_{m} M\right)$.

The profile coefficients influence the definition of the flux-balance parameter $\Gamma$, which expresses the ratio of buoyancy force to inertia (Morton 1959). As discussed in the introduction, $\Gamma=0$ for a pure jet and $\Gamma=1$ for a pure plume. In the far field, the first-order (velocity, buoyancy) and second-order (Reynolds stresses, buoyancy variance) statistics are fully self-similar (i.e. preserve shape upon rescaling radius and the quantity in consideration by the local characteristic scales $r_{m}, w_{m}$ and $b_{m}$ ), implying that the profile coefficients are therefore constant. In this case, (2.7)-(2.9) are consistent with the classical plume equations

$$
\frac{\mathrm{d} Q}{\mathrm{~d} z}=2 \alpha M^{1 / 2}, \quad \frac{\mathrm{d} M}{\mathrm{~d} z}=\frac{F_{E} Q}{M}, \quad \frac{\mathrm{d} F_{E}}{\mathrm{~d} z}=-N_{E}^{2} Q,
$$

where $F_{E}$ and $N_{E}$ are the effective buoyancy flux and frequency respectively, defined as

$$
F_{E} \equiv \frac{F}{\beta_{g} \theta_{m}}, \quad N_{E} \equiv \frac{N}{\left(\theta_{g} \beta_{g}\right)^{1 / 2}} .
$$

The connection with the standard plume equations is valuable because these have been widely applied to model problems of further complexity (e.g. the ventilation of buildings; Linden 1999). Equation $(2.13 a, b)$ indicates how effects from turbulence (via $\beta_{g}$ ) and differences in the shape of the buoyancy and the velocity profiles (via $\theta_{m}$ ) influence solutions to the classical plume equations and the practical applications to which they have been applied. For a pure plume in an unstratified environment, the solutions of $(2.12 a-c)$ are the power-law solutions (see, e.g., Turner 1979)

$$
Q(z)=\frac{6 \alpha_{p}}{5} M^{1 / 2} z, \quad M(z)=\left(\frac{9}{10}\right)^{2 / 3} \alpha_{p}^{2 / 3} F_{E}^{2 / 3} z^{4 / 3}, \quad F_{E}=\text { const. }
$$

where $\alpha_{p}$ is the entrainment coefficient for a pure plume. The definition of the fluxbalance parameter $\Gamma$ (Morton 1959) generalises to

$$
\Gamma=\frac{5 F_{E} Q^{2}}{8 \alpha_{p} M^{5 / 2}}=\frac{5 F Q^{2}}{8 \alpha_{p} \beta_{g} \theta_{m} M^{5 / 2}}=\frac{5}{8 \alpha_{p} \beta_{g}} R i .
$$


Here, the plume Richardson number $R i$, representative of the ratio of gravitational to inertial forcing, is defined as

$$
R i \equiv \frac{b_{m} r_{m}}{w_{m}^{2}}=\frac{F Q^{2}}{\theta_{m} M^{5 / 2}} .
$$

By construction, $\Gamma=1$ for a pure plume, as is clear from substituting the power-law solution $(2.14 a, b)$ into (2.15). The associated Richardson number is $R i_{p}=8 \alpha_{p} \beta_{g} / 5$; indeed, $\Gamma$ can be alternatively defined as $\Gamma=R i / R i_{p}$.

\section{The entrainment relation}

The system (2.7)-(2.10) comprises four equations with three dependent variables $(Q$, $M$ and $F$ ) and is thus overdetermined. However, the energy conservation equation (2.4) is derived from, and fully consistent with, the volume and momentum conservation equations (2.1) and (2.2). Integration over the radial direction cannot alter this property, and thus (2.10) must also be fully consistent with (2.7) and (2.8). This places a restriction on $\alpha$, which was introduced into (2.7) by invoking the entrainment hypothesis (1.1).

Using (2.7) as a definition of $\alpha$ and applying the product rule of differentiation to (2.10) and (2.8), it follows that

$$
\begin{aligned}
\alpha & \equiv \frac{1}{2 M^{1 / 2}} \frac{\mathrm{d} Q}{\mathrm{~d} z} \\
& =\frac{Q}{\beta_{g} M^{3 / 2}} \frac{\mathrm{d}}{\mathrm{d} z}\left(\beta_{g} M\right)-\frac{Q^{2}}{2 \gamma_{g} M^{5 / 2}} \frac{\mathrm{d}}{\mathrm{d} z}\left(\gamma_{g} \frac{M^{2}}{Q}\right)+\frac{Q}{2 M^{1 / 2}} \frac{\mathrm{d}}{\mathrm{d} z}\left(\log \frac{\gamma_{g}}{\beta_{g}^{2}}\right) \\
& =-\frac{\delta_{g}}{2 \gamma_{g}}+\left(\frac{1}{\beta_{g}}-\frac{\theta_{m}}{\gamma_{g}}\right) R i+\frac{Q}{2 M^{1 / 2}} \frac{\mathrm{d}}{\mathrm{d} z}\left(\log \frac{\gamma_{g}}{\beta_{g}^{2}}\right) .
\end{aligned}
$$

For an interpretation of the terms in (3.1), see $\S 4$. The entrainment relation (3.1) is a consistency requirement that is valid independently of model choices. This consistency requirement is valuable because entrainment models - which make particular assumptions regarding $\beta_{g}, \theta_{m}$ and $\gamma_{g}$ - have received criticism for not being applicable to specific flows, e.g. for clouds (Squires \& Turner 1962) or for reacting plumes (Ricou \& Spalding 1961; Hermanson \& Dimotakis 1989). Indeed, the attractiveness of (3.1) is that it is a relation with which all models for entrainment must be consistent. If a particular entrainment model is not in agreement with observations, it therefore becomes a relatively simple matter to pinpoint the cause of the discrepancy. The spreading rate $\mathrm{d} r_{m} / \mathrm{d} z$ is closely associated with, and is often used to infer, $\alpha$. It is therefore useful to establish how this quantity can be decomposed:

$$
\begin{aligned}
\frac{\mathrm{d} r_{m}}{\mathrm{~d} z} & =\frac{1}{M^{1 / 2}} \frac{\mathrm{d} Q}{\mathrm{~d} z}-\frac{Q}{2 M^{3 / 2}} \frac{\mathrm{d} M}{\mathrm{~d} z} \\
& =-\frac{\delta_{g}}{\gamma_{g}}+\frac{3}{2}\left(\frac{1}{\beta_{g}}-\frac{4}{3} \frac{\theta_{m}}{\gamma_{g}}\right) R i+\frac{Q}{M^{1 / 2}} \frac{\mathrm{d}}{\mathrm{d} z}\left(\log \frac{\gamma_{g}}{\beta_{g}^{3 / 2}}\right) .
\end{aligned}
$$

Table 1 contains a hierarchy of entrainment relations, which will be detailed below. The entrainment relation (3.1), hereafter called $\alpha$-F, includes the effects of 


$$
\begin{array}{lcc}
\text { Relation } & \text { Assumptions } & \alpha \\
\alpha \text {-F } & \text { None } & -\frac{\delta_{g}}{2 \gamma_{g}}+\left(\frac{1}{\beta_{g}}-\frac{\theta_{m}}{\gamma_{g}}\right) R i+\frac{Q}{2 M^{1 / 2}} \frac{\mathrm{d}}{\mathrm{d} z}\left(\log \frac{\gamma_{g}}{\beta_{g}^{2}}\right) \\
\alpha-\mathrm{M} & \text { Mean } & -\frac{\delta_{m}}{2 \gamma_{m}}+\left(1-\frac{\theta_{m}}{\gamma_{m}}\right) R i+\frac{Q}{2 M^{1 / 2}} \frac{\mathrm{d}}{\mathrm{d} z}\left(\log \gamma_{m}\right) \\
\alpha-\mathrm{S} & \text { Self-similar } & -\frac{\delta_{g}}{2 \gamma_{g}}+\left(\frac{1}{\beta_{g}}-\frac{\theta_{m}}{\gamma_{g}}\right) R i \\
\alpha-\mathrm{MS} & \text { Mean self-similar } & -\frac{\delta_{m}}{2 \gamma_{m}}+\left(1-\frac{\theta_{m}}{\gamma_{m}}\right) R i \\
\alpha-\mathrm{MSG} & \text { Gaussian, } \theta_{m}=1 & -\frac{3}{8} \delta_{m}+\frac{1}{4} R i
\end{array}
$$

TABLE 1. The hierarchy of entrainment relations. These relations are based on an integral or 'top-hat' description using definitions $(2.5 a-d)$ and $(2.6 a-c)$; for a conversion to a Gaussian or other description see appendix B.

mean quantities, turbulence and pressure. This entrainment relation makes minimal assumptions about the flow and will form the basis of all simplified entrainment relations described below. It is ideal for use in a diagnostic mode using data obtained from direct simulation, as was done in Craske \& van Reeuwijk (2015a).

Upon making the assumption that $\beta_{g}=\beta_{m}=1, \gamma_{g}=\gamma_{m}$ and $\delta_{g}=\delta_{m}$ (i.e. ignoring turbulence and pressure effects), the entrainment relation $\alpha-\mathrm{M},(3.4)$, is obtained. This is the entrainment relation that was derived by Kaminski et al. (2005), although it is not immediately obvious that $\alpha-\mathrm{M}$ is indeed consistent with their entrainment model; a detailed proof of the equivalence of the two models is provided in appendix A. In contrast to the relation derived by Kaminski et al. (2005), the 'similarity drift' term in $\alpha-\mathrm{M}$ (cf. third term), does not depend on the shape of the buoyancy profile via $\theta_{m}$ (see also Ezzamel et al. 2015), but only on $\gamma_{m}$, i.e. the shape of the velocity profile. The reason for this difference resides in the use of standard top-hat scales in this paper, see appendix $\mathrm{A}$ for details. Instead, variations in $\theta_{m}$ influence the second term in $\alpha$ $\mathrm{M}$ only, capturing the possible slow variation in the shape of the buoyancy profile discussed in Kaminski et al. (2005). This entrainment relation is ideal for examining the physics of turbulent plumes using experimental data. Indeed, many modern particle image velocimetry and laser Doppler anemometry systems can record high-frequency velocity fields, which, if augmented with appropriate buoyancy measurements, provide access to all of the required coefficients in $\alpha$-M. In complexity, the extension of the Kaminski entrainment relation by Ezzamel et al. (2015) sits between $\alpha-\mathrm{F}$ and $\alpha-\mathrm{M}$ and will not be laboured further here.

By assuming full self-similarity in the first- and second-order statistics, and therefore that $\beta_{m}, \gamma_{m}$ and $\delta_{m}$ are constants, the entrainment relation $\alpha$-MS is obtained, see (3.6). Formally, the only strict requirement of obtaining (3.6) from (3.4) is that $\gamma_{m}$ is constant. The relation $\alpha$-MS is the entrainment relation derived by Fox (1970), and describes the fundamental connection between the two main unknowns, $\delta_{m}$ and $\theta_{m}$, and the entrainment coefficient $\alpha$. Upon assuming that the profiles are Gaussian and that $\theta_{m}=1,(3.7)$ is obtained, which will be referred to as $\alpha$-MSG. The spreading 


$$
\begin{array}{lcc}
\text { Relation } & \text { Assumptions } & \mathrm{d} r_{m} / \mathrm{d} z \\
\alpha \text {-F } & \text { None } & -\frac{\delta_{g}}{\gamma_{g}}+\frac{3}{2}\left(\frac{1}{\beta_{g}}-\frac{4}{3} \frac{\theta_{m}}{\gamma_{g}}\right) R i+\frac{Q}{M^{1 / 2}} \frac{\mathrm{d}}{\mathrm{d} z}\left(\log \frac{\gamma_{g}}{\beta_{g}^{3 / 2}}\right) \\
\alpha-\mathrm{M} & \text { Mean } & -\frac{\delta_{m}}{\gamma_{m}}+\frac{3}{2}\left(1-\frac{4}{3} \frac{\theta_{m}}{\gamma_{m}}\right) R i+\frac{Q}{M^{1 / 2}} \frac{\mathrm{d}}{\mathrm{d} z}\left(\log \gamma_{m}\right) \\
\alpha-\mathrm{S} & \text { Self-similar } & -\frac{\delta_{g}}{\gamma_{g}}+\frac{3}{2}\left(\frac{1}{\beta_{g}}-\frac{4}{3} \frac{\theta_{m}}{\gamma_{g}}\right) R i \\
\alpha-\mathrm{MS} & \text { Mean self-similar } & -\frac{\delta_{m}}{\gamma_{m}}+\frac{3}{2}\left(1-\frac{4}{3} \frac{\theta_{m}}{\gamma_{m}}\right) R i \\
\alpha-\mathrm{MSG} & \text { Gaussian, } \theta_{m}=1 & -\frac{3}{4} \delta_{m}
\end{array}
$$

TABLE 2. The spreading rate $\mathrm{d} r_{m} / \mathrm{d} z$ associated with the entrainment relation. These relations are based on an integral or 'top-hat' description using definitions $(2.5 a-d)$ and $(2.6 a-c)$; for a conversion to a Gaussian or other description see appendix B.

rate associated with each entrainment relation is presented in table 2. One striking feature of the spreading rate equations is that under the realistic assumption that the far-field behaviour can be described by self-similar Gaussian profiles of equal width (relation $\alpha$-MSG), the $R i$-term associated with the net effect of buoyancy is identically zero, implying that the spreading rate is determined purely by the profile coefficient associated with the production of turbulence kinetic energy.

\section{Physics of entrainment}

In this section, we interpret the physical meaning of the three terms of the entrainment relation. The first term on the right-hand side of (3.3), $-\delta_{g} /\left(2 \gamma_{g}\right)$, is the ratio of the dimensionless turbulence production term $\delta_{g}$ and the dimensionless energy flux $\gamma_{g}$. It should be noted that $\delta_{g}<0$ under normal circumstances because the production of turbulence kinetic energy is a sink term in the equation for mean kinetic energy (2.10). For jets $(R i=0)$, this is the only non-zero term in the far field (Craske \& van Reeuwijk 2015a). For both pure jets and pure plumes, $\delta_{g}$ is dominated by $\delta_{m}$, the other production term $\delta_{f}$ and pressure redistribution term $\delta_{p}$ being comparatively small (Craske 2015; Craske \& van Reeuwijk 2015a).

The second term in (3.3) is the net effect of buoyancy on the entrainment coefficient, which we will discuss in depth in the next paragraphs. The third term is associated with streamwise changes in the shape of the velocity statistics through the profile coefficients $\beta_{g}$ and $\gamma_{g}$. These quantities are primarily associated with the mean flow, but also contain pressure and turbulence contributions, the latter being known to require a large distance to come to a full equilibrium (Wang \& Law 2002; Ezzamel et al. 2015). The third term is associated with the similarity drift discussed in Kaminski et al. (2005) and Carazzo et al. (2006), although as previously mentioned the present formulation does not contain contributions of $\theta_{m}$ because of the standard top-hat definitions of $r_{m}$ and $w_{m}$ used in this paper (see appendix A).

The entrainment relation $\alpha$-MS, which assumes full self-similarity and leading-order contributions only, will be used to further interpret the physics of turbulent 


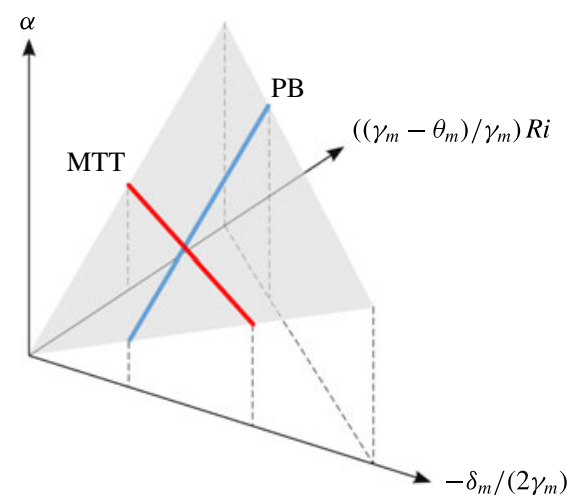

FIGURE 1. (Colour online) The far-field entrainment relation $\alpha$-MS, plotted together with the MTT entrainment model (fixed $\alpha$; red line) and the PB entrainment model (fixed $\delta_{m}$; blue line).

entrainment. As emphasised earlier, entrainment relations ensure consistency between volume, momentum and energy conservation at the integral level. Figure 1 shows the functional dependence of the entrainment coefficient $\alpha$ on the turbulence production contribution $-\delta_{m} /\left(2 \gamma_{m}\right)$ and net buoyancy $\left(1-\theta_{m} / \gamma_{m}\right) R i$ as a grey isosurface. Only points on this surface are physically realisable; therefore, models of entrainment must necessarily be defined on this surface. Two entrainment models associated with the MTT and PB plume theories, which form the subject of $\S 5$, are shown by red and blue lines respectively. The entrainment relation makes no assumption about how profile coefficients (e.g. $\delta_{m}, \gamma_{m}$ ) depend on properties of the flow such as the Richardson number $R i$, source conditions and local environmental conditions, for example. Indeed, the way in which entrainment depends on these aspects of the flow remains an open question, which is partially addressed for flows in an unstratified ambient in $\S 6$. The entrainment relation does, however, impose a fundamental consistency requirement linking the various model parameters, and therefore provides a necessary framework for the investigation of entrainment. In the far field, where profiles are assumed to be fully self-similar, profile coefficients will only depend on $R i$. For unstratified situations and for plumes with a constant buoyancy flux, the only cases for which $R i$ (and thus $\Gamma$ ) remains constant as a function of $z$ are a jet and a pure plume; it is, however, theoretically possible to achieve other constant- $R i$ solutions by considering, e.g., stratification, see $\$ 7$.

Within the $\alpha$-MS assumptions, the entrainment relation (3.6) follows from

$$
\alpha=\frac{r_{m}}{M} \frac{\mathrm{d} M}{\mathrm{~d} z}-\frac{r_{m}}{2 E} \frac{\mathrm{d} E}{\mathrm{~d} z},
$$

where $E=\gamma_{m} M^{2} / Q$ is the mean energy flux. The contribution of the normalised momentum flux $\left(r_{m} / M\right)(\mathrm{d} M / \mathrm{d} z)$ is the entrainment associated with the buoyancy force in the momentum equation, which evaluates to $R i$. The normalised energy-flux term $\left(r_{m} / E\right)(\mathrm{d} E / \mathrm{d} z)$ evaluates to $-\delta_{m} /\left(2 \gamma_{m}\right)-\left(\theta_{m} / \gamma_{m}\right) R i$. By using $M^{1 / 2}=Q / r_{m}$ in the definition of $\alpha,(4.1)$ can be written as

$$
\alpha=\frac{1}{2} \frac{\Delta Q}{Q}=\frac{\Delta M}{M}-\frac{1}{2} \frac{\Delta E}{E},
$$


where $\Delta Q=r_{m} \mathrm{~d} Q / \mathrm{d} z, \Delta M=r_{m} \mathrm{~d} M / \mathrm{d} z$ and $\Delta E=r_{m} \mathrm{~d} E / \mathrm{d} z$ represent changes over the characteristic length scale $r_{m}$. Thus, $\alpha$ can be interpreted as (half) the relative increase in $Q$ over a characteristic length $r_{m}$. The decomposition shows that an increase in the momentum flux $\Delta M>0$ will contribute positively to $\alpha$ while an increase in the energy flux $\Delta E>0$ contributes negatively. The balance of these two normalised fluxes depends sensitively on the profile coefficients. In a jet $\Delta M=0$ but $\Delta E<0$, because energy in the mean flow is converted to turbulence kinetic energy (cf. the first term on the right-hand side of (3.6)), implying that $\alpha>0$. The effect of buoyancy is somewhat more subtle. While buoyancy increases the momentum flux of the plume $(\Delta M>0)$, the buoyancy flux also increases the energy flux of the plume $(\Delta E>0)$, implying that buoyancy contributes both positively and negatively to $\alpha$ respectively. The net effect of buoyancy on $\alpha$ depends on the profile coefficients; for a Gaussian plume the net effect is positive, as is evident in (3.7).

Within the $\alpha$-MS assumptions, the explicit buoyancy contribution to $\alpha$ is purely associated with the mean flow, which suggests that a buoyant plume has an entrainment mechanism that does not directly rely on turbulence. To illustrate this it is instructive to perform a thought experiment and set $\delta_{m}=0, \theta_{m}=1$, i.e. we consider a plume that does not produce turbulence. The following two cases can be considered.

(1) A 'top-hat' plume with velocity profile $\bar{w}=w_{m} H\left(1-r / r_{m}\right)$ where $H$ is the Heaviside function, implying that $\gamma_{m}=1$. In this case, both $\Delta M>0$ and $\Delta E>0$ due to the buoyancy. However, as $\left(1-\theta_{m} / \gamma_{m}\right) R i=0$, the plume does not entrain, i.e. $\alpha=0$. The radius $r_{m}$ will decrease in order to satisfy momentum and energy conservation, cf. (3.11).

(2) A Gaussian plume with velocity profile $\bar{w}=2 w_{m} \exp \left(-2 r^{2} / r_{m}^{2}\right)$, implying that $\gamma_{m}=4 / 3$. Since $\gamma_{m}=4 / 3>1$, the value of $\Delta E / E$ is less negative for a Gaussian than for a top-hat plume. Consequently $\left(1-\theta_{m} / \gamma_{m}\right) R i>0$, implying that $\alpha>0$ : the Gaussian plume entrains in order to satisfy momentum and energy conservation. From (3.11) it follows that the solution is a column of fluid of constant radius.

In both cases considered here, the fluid accelerates as $\Delta M>0$ while the radius is either narrowing (top-hat plume) or remains constant (Gaussian plume). Without turbulence production, i.e. $\delta_{m}=0$, a top-hat plume is not able to entrain. However, a Gaussian plume is able to entrain even in the absence of turbulence. Although a Gaussian profile clearly requires turbulence to maintain its shape, the argument above demonstrates that buoyancy provides plumes with an entrainment mechanism not directly associated with turbulence.

\section{Entrainment models}

In this section, we look at the MTT and PB theories in the light of the Gaussian farfield entrainment relation $\alpha$-MSG. While the MTT theory does not explicitly assume that profiles are Gaussian, it can nevertheless be cast into the form of $\alpha$-MSG, as we will show. The PB theory assumes that $\delta_{m}$ takes a constant value $\delta_{P B}$, while the MTT model assumes that the entrainment coefficient $\alpha_{M T T}$ is a constant. These assumptions represent lines on the realisability surface of the entrainment relation, as shown in figure 1. It should be noted that the axes in this figure are not independent. Indeed, as argued in the previous section, $\alpha$ and $\delta_{m}$ are both expected to depend on $R i$ and, if non-equilibrium effects are included, also on $z$. In this light, the MTT and PB 
models form two limit cases: the MTT model assumes that $\alpha$ is independent of $R i$, while the PB model assumes that $\delta_{m}$ is independent of $R i$. Evidently, observations are required to determine under which circumstances the various models describe the physics accurately. We will examine the evidence for pure jets and plumes in an unstratified ambient in $\S 6$.

\subsection{The PB entrainment model}

Substitution of a constant value $\delta_{P B}$ into the entrainment relation (3.7) provides the following model for the entrainment coefficient:

$$
\alpha_{P B}(R i)=-\frac{3}{8} \delta_{P B}+\frac{1}{4} R i,
$$

or equivalently, in terms of the flux parameter $\Gamma$ (using $\beta_{g}=1$, consistent with the $\alpha$-MS relation),

$$
\alpha_{P B}(\Gamma)=-\frac{3}{8} \delta_{P B}+\frac{2}{5} \alpha_{p} \Gamma .
$$

For a pure jet $(\Gamma=0)$ it follows that $\alpha_{j}=-(3 / 8) \delta_{P B}$, implying that $\alpha_{P B}=\alpha_{j}+$ $(2 / 5) \alpha_{p} \Gamma$. By substituting the pure plume condition $\Gamma=1$ (for which by definition $\alpha_{P B}=\alpha_{p}$ ) it follows directly that

$$
\alpha_{p}=\frac{5}{3} \alpha_{j} .
$$

This remarkable prediction is mentioned in passing in List \& Imberger (1973, equation (25)) where an entrainment model is derived starting from straight-sidedness that is consistent with the entrainment model implied by the PB plume equations.

Here, we note that according to the PB model, the higher entrainment of the plume is a result not of buoyancy-enhanced turbulence production but of the entrainment associated with the mean flow. Substitution of (5.3) back into (5.2) results in the operational entrainment model (1.2). A striking feature of the PB model is that the solutions are straight-sided, as can be inferred from (3.12), which is a consequence of $\delta_{P B}$ being constant. Straight-sidedness is perhaps the most crucial assumption of the PB model and is a testable hypothesis. This is physically not realistic in the near field, where necking is often observed, and near the level of neutral buoyancy, where the plume spreads out. Indeed, in both cases one does not expect turbulence production to remain in proportion to $w_{m}^{3} r_{m}$.

\subsection{The MTT entrainment model}

Substitution of a constant value $\alpha_{M T T}$ for $\alpha$ into the entrainment relation (3.7) results in

$$
\delta_{M T T}(R i)=-\frac{8}{3} \alpha_{M T T}+\frac{2}{3} R i=-\frac{16}{15} \alpha_{M T T}\left(\frac{5}{2}-\Gamma\right) .
$$

It thus follows directly that the model predicts that pure plumes $(\Gamma=1)$ produce less turbulence than jets $(\Gamma=0)$. Moreover, according to the MTT entrainment model plumes are expected to be narrower than jets, since

$$
\frac{\mathrm{d} r_{m}}{\mathrm{~d} z}=2 \alpha_{M T T}-\frac{1}{2} R i=\frac{4 \alpha_{M T T}}{5}\left(\frac{5}{2}-\Gamma\right) .
$$

Equation (5.5), derived here via the entrainment relation, is consistent with van den Bremer \& Hunt (2010, equation (2.14)). This prediction is contrary to observations, which typically indicate that plumes have a slightly greater spreading rate than jets (e.g. List 1982). However, it should be noted that it is standard practice to choose a different value for $\alpha$ depending on whether one wishes to carry out jet or plume predictions, and the observed deficiency of the MTT entrainment model is therefore obscured. Nevertheless, for situations in which $R i$ varies as a function of $z$, e.g. for 
lazy/forced plumes in a neutral environment or plumes in stratified environments, one will have to decide on a single value for $\alpha$. The $R i$ dependence of the spreading rate in (5.5) is the reason why the MTT plume equations are able to predict plume necking in the near field for lazy plumes. Indeed, for $\Gamma>5 / 2, \delta_{M T T}$ changes sign and $\mathrm{d} r_{m} / \mathrm{d} z<0$. Similarly, the model predicts the plume spreading out to infinity at the neutral buoyancy level. The capability to capture necking and spreading is an extremely useful feature of the MTT model, although further work is needed to understand the extent to which the model faithfully represents the underlying physical processes.

\section{Pure jets and plumes in an unstratified environment}

In this section we investigate entrainment for pure jets and plumes in an unstratified environment using data available in the literature. We focus on $\alpha, \delta_{m}, \gamma_{m}$ and, in the case of a plume, $\theta_{m}$. Table 3 shows the values of these coefficients for the following studies: Papanicolaou \& List (1988, PL88); Panchapakesan \& Lumley (1993a, PL93); Shabbir \& George (1994, SG94); Wang \& Law (2002, WL02); Craske (2015, $R e=1600$, CvR15c); Craske \& van Reeuwijk (2015a, $R e=4815$, CvR15a) and Ezzamel et al. (2015, ESH15). Often, there is more than one way in which a coefficient can be inferred from a given data set, resulting in some systematic uncertainty in the estimates. For example, to estimate $\alpha$ from the direct numerical simulation data, Craske \& van Reeuwijk (2015a) compute $(\mathrm{d} Q / \mathrm{d} z) /\left(2 M^{1 / 2}\right)$ directly, whereas experimentalists (see, e.g., Wang \& Law 2002) typically prefer to infer $\alpha$ from the spreading rate of the plume by fitting a Gaussian curve to the profiles of mean velocity. Only in the case of fully self-similar Gaussian plumes are these two approaches equivalent. The cases selected were chosen because they provide the value of $\alpha$, the profiles of mean velocity, Reynolds stress and, in the case of a plume, mean buoyancy. This provides sufficient information to evaluate all terms in the entrainment relation $\alpha$-MS, (3.6). The standard procedure was to digitise the profiles and then to evaluate the coefficients numerically, converting to the scales used in this paper where required. Where possible, we made use of function fits of the data (WL02). The profile coefficients stated for ESH15 were calculated directly from the far-field source data and the values of $\alpha$ were determined directly from the spreading rate $\mathrm{d} r_{m} / \mathrm{d} z$ (P. Salizzoni, private communication). It should be noted that the values of $\alpha$ for the ESH15 dataset presented here are significantly larger than those presented in Ezzamel et al. (2015), which were estimated from the continuity equation. The underprediction is due to a systematic error associated with a small but significant co-flow (P. Salizzoni, private communication). The last four rows of table 3 (highlighted ${ }^{a}$ ) contain the converted Kaminski coefficients $A$ and $C$ (see appendix A). Here, we note that our estimate for $\delta_{m}$ in WL02 is lower than for $\mathrm{WL}^{a}{ }^{a}$. Having determined $\delta_{m}, \gamma_{m}$ and $\theta_{m}$, the indirect estimate $\alpha$-MS can be determined and compared with the direct estimate $\alpha$. We will first show that $\alpha$-MS provides a reasonably complete description of the entrainment physics and then investigate the contributions of the individual terms comprising $\alpha$-MS.

The last column in table 3 presents the absolute relative difference between the observed value of $\alpha$ and the estimate $\alpha$-MS. The two estimates generally agree to within $5 \%$ for the jet data, and to within $15 \%$ for the plume data. The ESH15 data have larger error bars, which are probably due to the large scatter in the Reynolds-stress profiles and the large value of $\theta_{m}$. Even though the entrainment relation $\alpha$-MS does not take into account contributions due to turbulence and pressure, the generally close agreement of the two estimates of $\alpha$ confirms that 


\begin{tabular}{lcccccccccc}
\hline Ref. & $\Gamma$ & $R i$ & $\alpha$ & $\delta_{m}$ & $\gamma_{m}$ & $\theta_{m}$ & $-\frac{\delta_{m}}{2 \gamma_{m}}$ & $\frac{\gamma_{m}-\theta_{m}}{\gamma_{m}} R i$ & $\alpha$-MS & $\frac{|\alpha-\alpha-\mathrm{MS}|}{\alpha}$ \\
& & & & & & & & & & \\
PL93a & 0.0 & 0.13 & 0.082 & -0.20 & 1.30 & - & 0.077 & - & 0.077 & 0.06 \\
WL02 & 0.0 & 0.12 & 0.075 & -0.21 & 1.33 & - & 0.079 & - & 0.079 & 0.05 \\
ESH15 & 0.0 & 0.12 & 0.072 & -0.21 & 1.33 & - & 0.079 & - & 0.079 & 0.10 \\
CvR15a & 0.0 & 0.11 & 0.069 & -0.19 & 1.30 & - & 0.073 & - & 0.073 & 0.06 \\
WL02 & 1.0 & 0.19 & 0.120 & -0.23 & 1.33 & 0.96 & 0.086 & 0.053 & 0.139 & 0.16 \\
ESH15 & 1.0 & 0.22 & 0.140 & -0.23 & 1.33 & 0.78 & 0.086 & 0.091 & 0.177 & 0.26 \\
CvR15c & 1.0 & 0.18 & 0.112 & -0.21 & 1.28 & 1.01 & 0.082 & 0.038 & 0.120 & 0.07 \\
PL88 & 1.0 & 0.19 & 0.120 & -0.20 & 1.36 & 0.93 & 0.074 & 0.060 & 0.134 & 0.12 \\
PL93b $^{a}$ & 0.5 & 0.15 & 0.095 & -0.17 & 1.50 & 0.79 & 0.057 & 0.071 & 0.128 & 0.35 \\
SG94 $^{a}$ & 1.0 & 0.24 & 0.150 & -0.26 & 1.19 & 1.08 & 0.109 & 0.022 & 0.131 & 0.13 \\
WL02 $^{a}$ & 1.0 & 0.19 & 0.120 & -0.27 & 1.39 & 0.96 & 0.097 & 0.059 & 0.156 & 0.30
\end{tabular}

TABLE 3. The observed entrainment coefficient and profile coefficients in jets and plumes. The data sources are Papanicolaou \& List (1988, PL88); Panchapakesan \& Lumley (1993a,b, PL93a, PL93b); Shabbir \& George (1994, SG94); Wang \& Law (2002, WL02); Craske (2015, CvR15c); Craske \& van Reeuwijk (2015a, CvR15a) and Ezzamel et al. (2015, ESH15).

${ }^{a}$ Data converted from Kaminski et al. (2005), Carazzo et al. (2006) using appendix A. ${ }^{b}$ Here, $\Gamma=0.5$ is assumed as a first-order approximation, because, as Carazzo et al. (2006) point out, the data from Panchapakesan \& Lumley (1993b, PL93b) pertain to an intermediate distance from a forced release, and therefore $0<\Gamma<1$.

$\alpha$-MS captures the primary entrainment mechanisms. Pressure redistribution and longitudinal turbulence production are responsible for the larger absolute relative difference between the two estimates of $\alpha$ for plumes than for jets, as can be inferred from direct numerical simulation data: the plume simulations of CvR15c indicate that $\delta_{g}=-0.20>-0.23=\delta_{m}$ due to contributions from $\delta_{f}$ and $\delta_{p}$, and with these contributions included the difference between the two estimates is less than $5 \%$.

A striking feature of the data in table 3 is that the value of $\delta_{m}$ is very similar for the jet and plume data, indicating that turbulence production is quite insensitive to $R i$. This in turn suggests that the PB entrainment model may appropriately capture the dynamics of jets and plumes in an unstratified environment when $0 \leqslant \Gamma \leqslant 1$. This hypothesis is further explored in figure 2, where the decomposed contributions to $\alpha$-MS of table 3 are presented graphically, together with the direct estimation of $\alpha$ (squares). The dashed lines represent the averages of $-\left\langle\delta_{m} /\left(2 \gamma_{m}\right)\right\rangle$ and $\langle\alpha-\mathrm{MS}\rangle$, where the operator $\langle\cdot\rangle$ denotes the average over the jet/plume data. It should be noted that the $\mathrm{WLO2}^{a}$ data were not included in the average to avoid double counting. Figure 2 reinforces the fact that $-\delta_{m} /\left(2 \gamma_{m}\right)$ is similar for a pure jet and a pure plume. Furthermore, for a pure plume the ratio of the average value of $\langle\alpha-\mathrm{MS}\rangle$ to $-\left\langle\delta_{m} /\left(2 \gamma_{m}\right)\right\rangle$ is within $5 \%$ of the value of $5 / 3$ predicted by the PB entrainment model.

An interesting implication of the fact that $\delta_{m}$ remains approximately constant is that the often-used argument (List 1982; Carazzo et al. 2006) of buoyancy-enhanced turbulence being the primary mechanism for the higher entrainment coefficient of plumes is not supported by the data. Indeed, enhanced turbulence levels would be expected to result in a larger value for $\delta_{m}$. Instead, it is a mean contribution to entrainment associated with the $R i$ dependence that is responsible for the higher entrainment coefficient. 


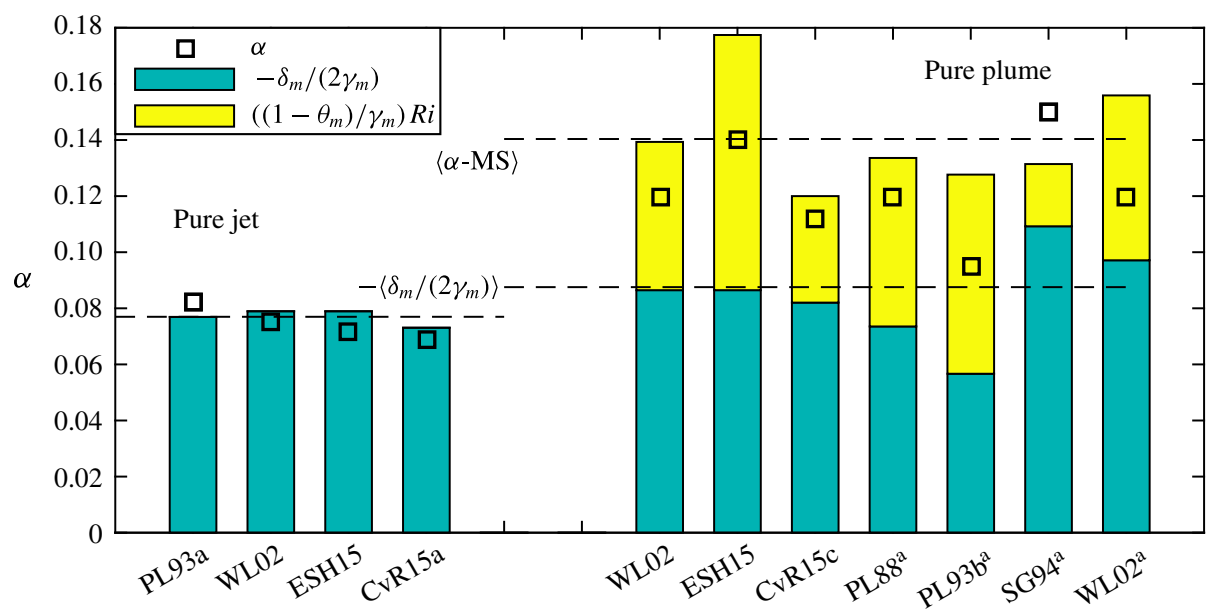

FIGURE 2. (Colour online) Individual terms of the entrainment relation $\alpha$-MS compared with a direct estimate of $\alpha$ (squares). The dashed lines are the averages of the individual terms of $\alpha$-MS, (3.6).

List \& Imberger (1973) and List (1982) observed that plumes and jets spread at a very similar rate, which, via (3.12), provides support for $\delta_{m}$ remaining approximately constant. Kaminski et al. (2005) calculate $\delta_{m}$ (which is proportional to their $C$ parameter, see appendix A) for pure plumes and then use the entrainment relation $\alpha$-MS to observe that this value is in close agreement with the jet data, and in accordance with previous observations that the Reynolds-stress profiles in jets and plumes are very similar (Panchapakesan \& Lumley 1993a; Wang \& Law 2002). Lastly, the forced plume experiments of Wang \& Law (2002), Matulka et al. (2014) and Ezzamel et al. (2015) show that the measured entrainment coefficient is in good agreement with the PB model.

In spite of the agreement that entrainment in forced plumes has with the PB model, the question of the most suitable parameterisation of entrainment in lazy plumes $(\Gamma>1)$ remains open. Bhat \& Narasimha (1996) report an observed narrowing in a heated (and consequently lazy) plume, which is broadly consistent with the MTT model for entrainment. However, in the absence of more comprehensive experimental and numerical data one is forced to accept the possibility that the true behaviour of the entrainment coefficient in lazy plumes might not coincide with either of the idealised models of PB and MTT. For lazy plumes one therefore assumes that $\alpha$ traces a more complicated curve on the realisability surface of the entrainment relation illustrated in figure 1.

\section{Entrainment at constant $R i$}

Further understanding of the way in which $\alpha$ depends on $R i$ requires knowledge of the dependence of $\delta_{g}, \gamma_{g}$ and $\theta_{g}$ on $R i$. Unfortunately, controlled observation of situations in which $R i$ is constant is difficult because (i), with the exception of pure jets and plumes, $R i$ typically varies in $z$ and (ii) changes in $R i$ are often accompanied by additional effects, such as the near-field development of a plume. To investigate the dependence of entrainment on $R i$ in isolation one would ideally conduct an experiment 
or simulation on a plume in which $R i$ remains constant. In this section we therefore present solutions, based on those originally obtained by Batchelor (1954) and Caulfield \& Woods (1998), for cases in which

$$
\frac{\mathrm{d} R i}{\mathrm{~d} z}=0,
$$

before discussing the ways in which (7.1) might be realised in practice. Substitution for $R i$ in (2.8) and (2.10) gives

$$
\begin{gathered}
\frac{\mathrm{d}}{\mathrm{d} z}\left(\beta_{g} M\right)=\frac{M^{3 / 2}}{Q} R i, \\
\frac{\mathrm{d}}{\mathrm{d} z}\left(\gamma_{g} \frac{M^{2}}{Q}\right)=\left(\delta_{g}+2 \theta_{m} R i\right) \frac{M^{5 / 2}}{Q^{2}},
\end{gathered}
$$

and, on expanding $R i$ in terms of $Q, M$ and $F$ using (2.16), (7.1) implies that

$$
\frac{\mathrm{d} F}{\mathrm{~d} z}=\theta_{m} R i \frac{\mathrm{d}}{\mathrm{d} z}\left(\frac{M^{5 / 2}}{Q^{2}}\right) .
$$

It should be noted that although we have not yet invoked an equation for buoyancy transport, (7.4) is a consistency requirement that determines the behaviour of $F$ for a given $R i$. We address the physical means of ensuring that $F$ behaves in this way in the following paragraph. Seeking solutions

$$
Q=Q_{0}\left(\frac{z}{z_{0}}\right)^{q}, \quad M=M_{0}\left(\frac{z}{z_{0}}\right)^{m}, \quad F=F_{0}\left(\frac{z}{z_{0}}\right)^{f},
$$

one finds

$$
q=3+\frac{a}{2}, \quad m=4+a, \quad f=4+\frac{3 a}{2},
$$

where, making use of $\alpha$-S to eliminate $\delta_{g}$ and $\gamma_{g}$,

$$
a \equiv \frac{6 R i / \beta_{g}-16 \alpha}{4 \alpha-R i / \beta_{g}} .
$$

When $R i=8 \alpha_{p} \beta_{g} / 5, a=-8 / 3$ and $q=5 / 3, m=4 / 3, f=0$, which is consistent with the classical self-similar plume solutions. Furthermore,

$$
Q_{0}=\left(\frac{z_{0}^{5} F_{0} R i^{4}}{\beta_{g}^{5} \theta_{m}(a+4)^{5}}\right)^{1 / 3}, \quad M_{0}=\left(\frac{z_{0}^{2} F_{0} R i}{\beta_{g}^{2} \theta_{m}(a+4)^{2}}\right)^{2 / 3}
$$

for prescribed values of $R i$ and $F_{0}$. The classical jet solutions correspond to the limit $a \rightarrow-4, F_{0} \rightarrow 0, R i \rightarrow 0$, in such a way that $Q_{0}$ and $M_{0}$ remain $O(1)$. Since $R i$ is constant, a notable feature of these solutions is that the spreading rate of the plume is a constant that depends on $R i$ and the profile coefficients. Observations of $\mathrm{d} r_{m} / \mathrm{d} z$ for a given $R i$ therefore provide a convenient way of establishing the relationship between the profile coefficients and $R i$ without direct measurement. Specifically, if a spreading rate $\mathrm{d} r_{m} / \mathrm{d} z$ is observed, one can use the implicit relation (see table 2)

$$
\frac{\mathrm{d} r_{m}}{\mathrm{~d} z}(R i)=-\frac{\delta_{g}(R i)}{\gamma_{g}(R i)}+\frac{3}{2}\left(\frac{1}{\beta_{g}(R i)}-\frac{4}{3} \frac{\theta_{m}(R i)}{\gamma_{g}(R i)}\right) R i
$$

to deduce the possible dependence of the profile coefficients on Ri. If, in addition, the volume flux is measured, then one can couple (7.9) with the relation for $\alpha$ to further 
restrict the possible form of dependence on $R i$ that the profile coefficients might have. In this way it is possible to deduce higher-order aspects of the flow by measuring lower-order bulk quantities.

From a practical perspective it is necessary to consider how a constant- $R i$ flow could be realised in the laboratory or in a numerical simulation. To this end, we assume that the buoyancy flux in the plume can be modified either directly via a heat source $S(z)$ or indirectly via a stratified environment with buoyancy frequency $N(z)$. With a heat source included, the conservation equation for the buoyancy flux becomes (cf. (2.9))

$$
\frac{\mathrm{d} F}{\mathrm{~d} z}=\frac{f F_{0}}{z}\left(\frac{z}{z_{0}}\right)^{f}=-\frac{\theta_{m}}{\theta_{g}} N^{2} Q+S, \quad \text { where } N^{2} \equiv N_{0}^{2}\left(\frac{z}{z_{0}}\right)^{a} .
$$

When $N=0$ (an unstratified environment), the power-law behaviour can be obtained by using a buoyancy source $S=f F / z$, where $F$ (see (7.5)) and $f$ (see (7.6)) are known functions of $R i$, the special case of constant $S$ having been examined by Hunt \& Kaye (2005). In a numerical simulation, a source of buoyancy of this kind can be imposed locally by forcing the buoyancy $b$ by an amount $f \bar{w} \bar{b} / z$. Experimentally, this could be realised by making the plume fluid conductive and subjecting it to a potential difference (see Bhat \& Narasimha 1996; Agrawal \& Prasad 2004). However, great care would be required to ensure that the radial dependence of the forcing function was fully understood, as it would most likely influence parameters such as $\delta_{g}$ and $\theta_{g}$.

Alternatively, a forcing in the buoyancy equation can be achieved by imposing a stratification in the ambient $\left(N^{2} \neq 0\right)$, resulting in

$$
Q_{0}=-\mathrm{i}\left(\frac{N_{0}^{2}}{f m \theta_{g} \beta_{g}}\right)^{1 / 2} \frac{4 \alpha^{2} z_{0}^{3}}{q^{2}}, \quad M_{0}=-\frac{4 \alpha^{2} N_{0}^{2} z_{0}^{4}}{f m q^{2} \theta_{g} \beta_{g}}, \quad F_{0}=-\frac{\theta_{m}}{\theta_{g}} \frac{N_{0}^{2} z_{0}}{f} Q_{0} . \quad(7.11 a-c)
$$

The condition that $Q_{0}$ and $F_{0}$ must remain real-valued can be satisfied in two ways: (1) $N_{0}^{2}<0$ and $f>0$, implying that $a>-8 / 3$, i.e. an increasing buoyancy flux in an unstable stratification (Batchelor 1954); or (2) $N_{0}^{2}>0$ and $f<0$, implying that $-4<a<-8 / 3$, i.e. a decreasing buoyancy flux in a stable stratification (Caulfield \& Woods 1998). While it is typically assumed that $\alpha$ is constant in these solutions, here $\alpha$ depends on $R i$ and therefore on $a$ :

$$
\alpha=-\frac{\delta_{g}}{2 \gamma_{g}}\left(4\left(\frac{a+4}{a+6}\right)\left(\frac{\theta_{m}}{\gamma_{g} \beta_{g}}-1\right)+1\right)^{-1} .
$$

Equation (7.12) therefore relates the scaling associated with an ambient stratification to the entrainment coefficient, subject to knowledge of the profile coefficients. When $a>$ $-8 / 3$, the exponent $f>0$, implying that the buoyancy flux increases with height. The plumes that these solutions describe develop in the absence of a heat source and were first identified by Batchelor (1954). They are lazy plumes because $a>-8 / 3$ implies that $\Gamma>1$. However, it is clear that the unstable stratification required for this regime would be difficult to realise in practice and that the methods of heating the plume described above provide a more practical means of investigating lazy plumes. Indeed, the use of a stratification is best suited to the investigation of forced plumes, which are obtained over the interval $-4<a<-8 / 3$, for which $f<0$ and $0<\Gamma<1$. These solutions correspond to a stratification that decays rapidly enough to maintain positive buoyancy flux in the plume, in spite of the fact that $f<0$ (Caulfield \& Woods 1998). Pure plume and jet solutions correspond to limits $f \rightarrow 0, z_{0} \rightarrow 0$ with $f^{3 / 8} / z_{0}=O(1)$ and $m \rightarrow 0, z_{0} \rightarrow 0$ with $m^{1 / 4} / z_{0}=O(1)$ respectively (see Caulfield \& Woods 1998 , for further details). 
Constant $R i$ similarity solutions provide a useful means of investigating entrainment in plumes because they are not restricted to the near field. Moreover, the freedom to modify $R i$ via an ambient stratification or a direct source of heating, or both, is valuable, because the resulting profile coefficients may well depend on the particular method employed.

\section{Concluding remarks}

This paper focused on the restrictions imposed upon the entrainment coefficient $\alpha$ by the conservation equation of mean kinetic energy. By integrating the Reynoldsaveraged axisymmetric conservation equation for volume, streamwise momentum, buoyancy and mean kinetic energy over the radius $r$, four coupled ODEs were obtained with three independent variables, $Q, M$ and $F$. From the requirement that all four equations must be satisfied simultaneously, a restriction on the entrainment coefficient $\alpha$ was obtained, which was referred to as an entrainment relation. The entrainment relation decomposes $\alpha$ into contributions due to turbulence production, buoyancy and changes in the profile coefficients. Entrainment due to buoyancy is primarily associated with mean-flow entrainment, i.e. turbulence does not play a direct role. As the normalised momentum- and energy-flux divergences contribute oppositely to entrainment, the shapes of the velocity and buoyancy profiles are a crucial factor in determining the net effect of buoyancy. The entrainment relation generalises previous entrainment relations that only incorporated mean-flow components (Kaminski et al. 2005) and fully self-similar mean profiles (Fox 1970). The resulting hierarchy of entrainment relations is summarised in table 1.

The entrainment relation, which is a physical constraint upon $\alpha$, must be considered separately from an entrainment model. Indeed, an entrainment model parameterises the a priori unknown dependence of $\delta_{g}, \gamma_{g}$ and $\theta_{m}$ on the plume Richardson number $R i$ in order to be able to provide predictions. The entrainment models implied by the plume theories of Priestley \& Ball (1955) and Morton et al. (1956) were reviewed in $\S 5$.

An analysis of the literature documenting laboratory experiments and simulations of pure jets and plumes in unstratified environments revealed that, to leading order, the PB entrainment model (1.2) provides an appropriate description of the physics, as was previously observed indirectly by Fox (1970), List \& Imberger (1973), Wang \& Law (2002) and Ezzamel et al. (2015). This implies that the assumption made in Priestley $\&$ Ball (1955) that the profile coefficient associated with the production of turbulence kinetic energy $\delta_{m}$ does not depend of the flux parameter $\Gamma$ is approximately valid. The fact that $\delta_{m}$ takes approximately the same value for a jet and a plume implies that the enhanced entrainment is due to buoyancy effects associated with the mean flow, rather than buoyancy-enhanced turbulence, and results in the value of $\alpha$ for a pure plume in an unstratified environment being a factor of $5 / 3$ larger than for a jet.

The MTT plume equations are used widely in engineering and the atmospheric sciences, often being incorporated in larger systems of further complexity (see, e.g., Linden 1999). One therefore questions whether the findings of this paper warrant a change from the constant- $\alpha$ MTT model to the variable- $\alpha$ PB model. In this regard we wish to point out that the current practice of employing a different value of $\alpha$ depending on whether one is dealing with a jet or a plume is equivalent to using the PB model. Thus, the implicit use of the PB model is perhaps more widespread than one might estimate from the literature. As described in the present paper, in the PB model the two different values of $\alpha$, pertaining to jets and plumes, emerge naturally from explicit assumptions about the physics. Consequently, unlike the MTT model, the PB model can be used to simulate the behaviour of forced plumes, in which $\alpha$ 
varies continuously. Indeed, one can employ the PB model to understand aspects of lazy plumes, although the precise influence of turbulent mixing and non-Boussinesq effects on entrainment in lazy plumes requires further investigation. In this regard our hope is that the information in this paper provides a useful framework and rigorous starting point for further study.

While the entrainment relation accounts for variations in $\alpha$ due to buoyancy and changes in profile shapes, $\alpha$ will retain a large degree of scatter due to additional uncertainties. Indeed, differences in the experimental apparatus and the inflow conditions remain an important factor, particularly relatively close to the source where turbulent quantities are still developing and a fully self-similar state has not yet been reached. It is likely that the reported variation in $\alpha$ is partially caused by differences in the large-scale structure of the turbulence, which can persist for extended periods of time (Redford et al. 2012).

The entrainment relation provides a diagnostic framework to systematically study entrainment. One interesting avenue of further research is to use the entrainment relation to study entrainment in the near field, where changes in profile shape and turbulence levels will be pronounced. Furthermore, an exploration of constant-Ri lazy and forced plumes, as described in $\S 7$, would allow for a systematic study of the dependence of the profile coefficients $\delta_{g}, \gamma_{g}$ and $\theta_{g}$ upon $R i$.

\section{Acknowledgements}

M.v.R. would like to thank P. Salizzoni for fruitful conversations about turbulent entrainment, experimental methods and detailed comments on the paper. In addition, J.C. gratefully acknowledges funding from the Engineering and Physical Sciences Research Council (EPSRC) under grant no. EP/J500239/1.

\section{Appendix A. Relation to Kaminski et al. (2005)}

In this appendix we demonstrate the one-to-one correspondence between the decomposition of the entrainment coefficient in (3.1) and that of Kaminski et al. (2005). To do this we will relate the characteristic scales for velocity, radius and buoyancy, $w_{m}, r_{m}$ and $b_{m}$ respectively, used in this paper, to the respective scales $\hat{w}$, $\hat{r}$ and $\hat{b}$ used by Kaminski et al. (2005, denoted $w_{m}, b_{m}$ and $g_{m}^{\prime}$ in their notation respectively). Products of the characteristic scales are equal to the various integrals appearing in the problem:

$$
\begin{aligned}
& \text { (this paper) } \\
& w_{m} r_{m}^{2}=2 \int_{0}^{\infty} \bar{w} r \mathrm{~d} r=2 \hat{w} \hat{r}^{2} \int_{0}^{\infty} f r_{*} \mathrm{~d} r_{*}=2 \hat{w} \hat{r}^{2} I_{0}, \\
& \theta_{m} w_{m} b_{m} r_{m}^{2}=2 \int_{0}^{\infty} \bar{w} \bar{b} r \mathrm{~d} r=2 \hat{w} \hat{b} \hat{r}^{2} \int_{0}^{\infty} f h r_{*} \mathrm{~d} r_{*}=2 \hat{w} \hat{b} \hat{r}^{2} I_{1}, \\
& b_{m} r_{m}^{2}=2 \int_{0}^{\infty} \bar{b} r \mathrm{~d} r=2 \hat{b} \hat{r}^{2} \int_{0}^{\infty} h r_{*} \mathrm{~d} r_{*}=2 \hat{b} \hat{r}^{2} I_{2}, \\
& w_{m}^{2} r_{m}^{2}=2 \int_{0}^{\infty} \bar{w}^{2} r \mathrm{~d} r=2 \hat{w}^{2} \hat{r}^{2} \int_{0}^{\infty} f^{2} r_{*} \mathrm{~d} r_{*}=2 \hat{w}^{2} \hat{r}^{2} I_{3} \text {, } \\
& \gamma_{m} w_{m}^{3} r_{m}^{2}=2 \int_{0}^{\infty} r \bar{w}^{3} \mathrm{~d} r=2 \hat{w}^{3} \hat{r}^{2} \int_{0}^{\infty} f^{3} r_{*} \mathrm{~d} r_{*}=2 \hat{w}^{3} \hat{r}^{2} I_{4}, \\
& \delta_{m} w_{m}^{3} r_{m}=4 \int_{0}^{\infty} r \overline{w^{\prime} u^{\prime}} \frac{\partial \bar{w}}{\partial r} \mathrm{~d} r=-2 \hat{w}^{3} \hat{r} \int_{0}^{\infty} j \frac{\partial f}{\partial r_{*}} r_{*} \mathrm{~d} r_{*}=-2 \hat{w}^{3} \hat{r} I_{5} .
\end{aligned}
$$


Here, the functions $f, h$ and $j$ are dimensionless radial profiles of velocity, buoyancy and Reynolds stress, and $r_{*}$ is a dimensionless radial coordinate. Equating the leftmost and right-most terms results in

$$
\begin{gathered}
I_{0}=\frac{1}{2} \frac{w_{m} r_{m}^{2}}{\hat{w} \hat{r}^{2}}, \quad I_{1}=\frac{\theta_{m}}{2} \frac{w_{m} b_{m} r_{m}^{2}}{\hat{w} \hat{b} \hat{r}^{2}}, \\
I_{2}=\frac{1}{2} \frac{b_{m} r_{m}^{2}}{\hat{b} \hat{r}^{2}}, \quad I_{3}=\frac{1}{2} \frac{w_{m}^{2} r_{m}^{2}}{\hat{w}^{2} \hat{r}^{2}}, \\
I_{4}=\frac{\gamma_{m}}{2} \frac{w_{m}^{3} r_{m}^{2}}{\hat{w}^{3} \hat{r}^{2}}, \quad I_{5}=-\frac{\delta_{m}}{2} \frac{w_{m}^{3} r_{m}}{\hat{w}^{3} \hat{r}} .
\end{gathered}
$$

Kaminski et al. (2005) work in terms of 'top-hat' variables $R, W$ and $G^{\prime}$ for velocity, radius and buoyancy respectively, defined according to

$$
R^{2} W G^{\prime}=\hat{w} \hat{b} \hat{r}^{2} I_{1}, \quad R^{2} G^{\prime}=\hat{b} \hat{r}^{2} I_{2}, \quad R^{2} W^{2}=\hat{w}^{2} \hat{r}^{2} I_{3} .
$$

Therefore, using (A 7) and (A 8) we find that

$$
G^{\prime}=b_{m} \theta_{m}^{2}, \quad R=\frac{r_{m}}{\sqrt{2} \theta_{m}}, \quad W=\theta_{m} w_{m} .
$$

From (A 11) it is evident that Kaminski et al. (2005) base their top-hat radius on the widths of both the velocity profile and the buoyancy profile.

The entrainment coefficient $\alpha_{e}$ used by Kaminski et al. (2005) is defined via

$$
\frac{\mathrm{d}}{\mathrm{d} z}\left(R^{2} W\right)=2 \alpha_{e} R W,
$$

where

$$
\alpha_{e}=\frac{1}{2} C+\frac{G^{\prime} R}{W^{2}}\left(1-\frac{1}{A}\right)+\frac{1}{2} R \frac{\mathrm{d}}{\mathrm{d} z} \log A
$$

and

$$
A \equiv \frac{I_{2} I_{4}}{I_{1} I_{3}}, \quad C \equiv \frac{I_{2} I_{3}^{1 / 2} I_{5}}{I_{1} I_{4}} .
$$

Substitution for $R$ and $W$ in (A 12) reveals the relationship between $\alpha_{e}$ and the $\alpha$ used in this paper:

$$
\frac{\mathrm{d} Q}{\mathrm{~d} z}=2 \underbrace{\left(\sqrt{2} \theta_{m} \alpha_{e}+\frac{r_{m}}{2} \frac{\mathrm{d}}{\mathrm{d} z} \log \theta_{m}\right)}_{\alpha} M^{1 / 2} .
$$

Furthermore, in terms of $\delta_{m}, \gamma_{m}$ and $\theta_{m}$, it follows that $A=\gamma_{m} / \theta_{m}$ and $C=$ $-\delta_{m} /\left(\sqrt{2} \theta_{m} \gamma_{m}\right)$. Substitution in (A 13) results in

$$
\alpha=-\frac{\delta_{m}}{2 \gamma_{m}}+\frac{\gamma_{m}-\theta_{m}}{\gamma_{m}} R i+\frac{r_{m}}{2} \frac{\mathrm{d}}{\mathrm{d} z} \log \gamma_{m},
$$

which is identical to the entrainment relation $\alpha-\mathrm{M}$, (3.4).

There are two advantages of (A 16) compared with (A 13). First, the effects of 'similarity drift' between the profiles of buoyancy and velocity are accounted for in a clean manner in the second term on the right-hand side of (A 16), outside derivatives. Second, (A 16) can be applied to jets $(R i=0)$ without difficulty. 


\section{Appendix B. Conversion to other flavours of the plume equations}

Perhaps one of the greatest complications in accessing the plume literature is the large number of different forms in which the plume equations can be presented, the most popular of which are the top-hat and Gaussian descriptions. The descriptions are all closely related (and do not make a statement about the actual shape of the radial profiles), with coefficients appearing in the equations in different locations. This is most evident in the value of the entrainment coefficient, which is a factor of $\sqrt{2}$ larger for a top-hat description than for a Gaussian description. The aim of this section is to demonstrate how entrainment models can be incorporated in existing implementations of the MTT plume equations.

The results presented in this paper are consistently based on integral quantities in order not to make an explicit assumption about profile shapes. Thus, in order to translate the entrainment models presented in this paper one should relate the dependent variables of the model under consideration to the fundamental integral quantities defined in $\S 2$. As an example, consider a model based explicitly on profiles of the form

$$
\bar{w}(r, z)=\hat{w} f_{w}\left(\frac{r}{\hat{r}}\right), \quad \bar{b}(r, z)=\hat{b} f_{b}\left(\frac{r}{\hat{r}}\right),
$$

where $r / \hat{r} \equiv \eta$ is a similarity variable and $\hat{w}, \hat{b}$ and $\hat{r}$ are the velocity amplitude, buoyancy amplitude and typical radius respectively. Associated with a similarity function $f_{\chi}$, where $\chi$ is a dependent variable, is a profile coefficient that will be denoted

$$
I_{\chi} \equiv \int_{0}^{\infty} f_{\chi} \eta \mathrm{d} \eta \text {. }
$$

Using this notation, the key integrals $Q, M, B$ and $F$ can be written as

$$
\begin{gathered}
Q=2 \int_{0}^{\infty} \bar{w} r \mathrm{~d} r=2 \hat{w} \hat{r}^{2} I_{w}, \quad M=2 \int_{0}^{\infty} \bar{w}^{2} r \mathrm{~d} r=2 \hat{w}^{2} \hat{r}^{2} I_{w w}, \\
B=2 \int_{0}^{\infty} \bar{b} r \mathrm{~d} r=2 \hat{b} \hat{r}^{2} I_{b}, \quad F=2 \int_{0}^{\infty} \bar{w} \bar{b} r \mathrm{~d} r=2 \hat{w} \hat{b} \hat{r}^{2} I_{w b},
\end{gathered}
$$

leading to the following relations for derived quantities:

$$
\begin{gathered}
r_{m} \equiv \frac{Q}{M^{1 / 2}}=\frac{\sqrt{2} I_{w}}{\sqrt{I_{w w}}} \hat{r}, \quad w_{m} \equiv \frac{M}{Q}=\frac{I_{w w}}{I_{w}} \hat{w}, \quad b_{m} \equiv \frac{B M}{Q^{2}}=\frac{I_{b} I_{w w}}{I_{w}^{2}} \hat{b}, \\
R i=\frac{\sqrt{2} I_{w} I_{b}}{I_{w w}^{3 / 2}} \frac{\hat{b} \hat{r}}{\hat{w}^{2}}, \quad-(r u)_{r=\infty}=2 \alpha M^{1 / 2}=2 \underbrace{\alpha \sqrt{2 I_{w w}}}_{\hat{\alpha}} \hat{w} \hat{r} .
\end{gathered}
$$

Upon substituting the Gaussian profiles $f_{w}=\exp \left(-r^{2} / \hat{r}^{2}\right)$ and $f_{b}=\exp \left(-r^{2} /(\lambda \hat{r})^{2}\right)$, where $\lambda \hat{r}$ is the characteristic width of the buoyancy profile, it follows that $I_{w}=1 / 2$, $I_{w w}=1 / 4, I_{b}=\lambda^{2} / 2$ and $I_{w b}=\lambda^{2} /\left(2\left(\lambda^{2}+1\right)\right)$. This implies that for Gaussian profiles

$$
r_{m}=\sqrt{2} \hat{r}, \quad w_{m}=\frac{1}{2} \hat{w}, \quad b_{m}=\frac{\lambda^{2}}{2} \hat{b}, \quad R i=2 \sqrt{2} \lambda^{2} \frac{\hat{b} \hat{r}}{\hat{w}^{2}}, \quad \alpha=\sqrt{2} \hat{\alpha}, \quad(\mathrm{B} 7 a-e)
$$


and thus the entrainment relation $\alpha$-MS is given by

$$
\hat{\alpha}=-\frac{\delta_{m}}{2^{3 / 2} \gamma_{m}}+2 \frac{2-\theta_{m}}{\theta_{m}} \frac{\gamma_{m}-\theta_{m}}{\gamma_{m}} \frac{\hat{b} \hat{r}}{\hat{w}^{2}},
$$

where $\delta_{m}, \gamma_{m}$ and $\theta_{m}$ are provided by the entrainment model.

\section{REFERENCES}

Agrawal, A. \& Prasad, A. K. 2004 Evolution of a turbulent jet subjected to volumetric heating. J. Fluid Mech. 511, 95-123.

BAtchelor, G. K. 1954 Heat convection and buoyancy effects in fluids. Q. J. R. Meteorol. Soc. 80 (345), 339-358.

Bhat, G. S. \& Narasimha, R. 1996 A volumetrically heated jet: large-eddy structure and entrainment characteristics. J. Fluid Mech. 325, 303-330.

VAN DEN BREMER, T. S.\& HUNT, G. R. 2010 Universal solutions for Boussinesq and non-Boussinesq plumes. J. Fluid Mech. 644, 165-192.

Carazzo, G., Kaminski, E. \& TAit, S. 2006 The route to self-similarity in turbulent jets and plumes. J. Fluid Mech. 547, 137-148.

Caulfield, C. P. \& Woods, A. W. 1998 Turbulent gravitational convection from a point source in a non-uniformly stratified environment. J. Fluid Mech. 360, 229-248.

Craske, J. 2015 Unsteady turbulent jets and plumes. PhD thesis, Imperial College London.

Craske, J. \& Van ReeuwiJK, M. $2015 a$ Energy dispersion in turbulent jets. Part 1. Direct simulation of steady and unsteady jets. J. Fluid Mech. 763, 500-537.

Craske, J. \& VAN ReEuWiJK, M. $2015 b$ Energy dispersion in turbulent jets. Part 2. A robust model for unsteady jets. J. Fluid Mech. 763, 538-566.

Delichatsios, M. A. 1979 Time similarity analysis of unsteady buoyant plumes in neutral surroundings. J. Fluid Mech. 93, 241-250.

Ezzamel, A., Salizzoni, P. \& Hunt, G. R. 2015 Dynamical variability of axisymmetric buoyant plumes. J. Fluid Mech. 765, 576-611.

FAnNELOP, T. K. \& WeBBeR, D. M. 2003 On buoyant plumes rising from area sources in a calm environment. J. Fluid Mech. 497, 319-334.

Fischer, H. B., List, E. J., Koh, R. C., Imberger, J. \& Brooks, N. H. 1979 Mixing in Inland and Coastal Waters. Academic.

Fox, D. G. 1970 Forced plume in a stratified fluid. J. Geophys. Res. 75 (33), 6818-6835.

George, W. K. 1989 The self-preservation of turbulent flows and its relation to initial conditions and coherent structures. In Advances in Turbulence (ed. R. E. A. Arndt \& W. K. George), pp. 39-73. Hemisphere Publishing Corp., New York.

Hermanson, J. C. \& Dimotakis, P. E. 1989 Effects of heat release in a turbulent, reacting shear layer. J. Fluid Mech. 199, 333-375.

Hunt, G. R. \& KaYe, N. B. 2005 Lazy plumes. J. Fluid Mech. 533, 329-338.

Hunt, G. R. \& VAN DEN BREMER, T. S. 2011 Classical plume theory: 1937-2010 and beyond. IMA J. Appl. Maths 76, 424-448.

Kaminski, E., Tait, S. \& CARAzzo, G. 2005 Turbulent entrainment in jets with arbitrary buoyancy. J. Fluid Mech. 526, 361-376.

Linden, P. F. 1999 The fluid mechanics of natural ventilation. Annu. Rev. Fluid Mech. 31, 201-238.

List, E. J. 1982 Turbulent jets and plumes. Annu. Rev. Fluid Mech. 14, 189-212.

List, E. J. \& Imberger, J. 1973 Turbulent entrainment in buoyant jets and plumes. J. Hydraul. Div. ASCE 99, 1461-1474.

Matulka, A., Lopez, P., Redondo, J. M. \& TARquis, A. 2014 On the entrainment coefficient in a forced plume: quantitative effects of source parameters. Nonlinear Process. Geophys. 21, 269-278.

Morton, B. R. 1959 Forced plumes. J. Fluid Mech. 5, 151-163. 
Morton, B. R. 1971 The choice of conservation equations for plume models. J. Geophys. Res. 30, 7409-7416.

Morton, B. R., TAYlor, G. I. \& Turner, J. S. 1956 Turbulent gravitational convection from maintained and instantaneous sources. Proc. R. Soc. Lond. A 234, 1-23.

Panchapakesan, N. R. \& Lumley, J. L. 1993a Turbulence measurements in axisymmetric jets of air and helium. Part 1. Air jet. J. Fluid Mech. 246, 197-223.

Panchapakesan, N. R. \& Lumley, J. L. $1993 b$ Turbulence measurements in axisymmetric jets of air and helium. Part 2. Helium jet. J. Fluid Mech. 246, 225-247.

PAPANicolaOU, P. N. \& List, E. J. 1988 Investigations of round vertical turbulent buoyant jets. J. Fluid Mech. 195, 341-391.

Priestley, C. H. B. \& BALL, F. K. 1955 Continuous convection from an isolated source of heat. Q. J. R. Meteorol. Soc. 81, 144-157.

Redford, J. A., Castro, I. P. \& Coleman, G. N. 2012 On the universality of turbulent axisymmetric wakes. J. Fluid Mech. 710, 419-452.

Ricou, F. P. \& SpAlding, D. B. 1961 Measurements of entrainment by axisymmetrical turbulent jets. J. Fluid Mech. 11 (01), 21-32.

Scase, M. M., Caulfield, C. P., Dalziel, S. B.\& Hunt, J. C. R. 2006 Time-dependent plumes and jets with decreasing source strengths. J. Fluid Mech. 563, 443-461.

Scase, M. M. \& Hewitt, R. E. 2012 Unsteady turbulent plume models. J. Fluid Mech. 697, $455-480$.

Shabbir, A. \& George, W. K. 1994 Experiments on a round turbulent buoyant plume. J. Fluid Mech. 275, 1-32.

Squires, P. \& TURner, J. S. 1962 An entraining jet model for cumulo-nimbus updraughts. Tellus 14 (4), 422-434.

Telford, J. W. 1966 The convective mechanism in clear air. J. Atmos. Sci. 23 (6), 652-666.

Tennekes, H. \& Lumley, J. L. 1972 A First Course in Turbulence. MIT Press.

TURner, J. S. 1979 Buoyancy Effects in Fluids. Cambridge University Press.

TURNER, J. S. 1986 Turbulent entrainment: the development of the entrainment assumption, and its application to geophysical flows. J. Fluid Mech. 173, 431-471.

Vul'Fson, A. N. \& BORODIN, O. O. 2001 Self-similar propagation regimes of a nonstationary high-temperature convective jet in the adiabatic atmosphere. J. Appl. Mech. Tech. Phys. 42, 255-261.

WANG, H. \& LAW, A. W.-K. 2002 Second-order integral model for a round turbulent buoyant jet. J. Fluid Mech. 459, 397-428.

YU, H. Z. 1990 Transient plume influence in measurement of convective heat release rates of fast-growing fires using a large-scale fire products collector. Trans. ASME J. Heat Transfer 112 (1), 186-191. 